\title{
The Casimir Operators of Inhomogeneous Groups
}

\author{
M. Chaichian ${ }^{1}$, A. P. Demichev ${ }^{2}$, and N. F. Nelipa ${ }^{2}$ \\ 1 Department of High Energy Physics, University of Helsinki, SF-00170 Helsinki, Finland \\ 2 Institute of Nuclear Physics, Moscow State University, Moscow, USSR
}

\begin{abstract}
We have found the number of invariant operators for the inhomogeneous groups $\operatorname{IGL}(n, R), \operatorname{ISL}(n, R), \operatorname{ISO}(p, q), \operatorname{IU}(p, q), \operatorname{ISU}(p, q)$, $\operatorname{ISp}(2 n)$, i.e. the inhomogeneous groups with the classical homogeneous subgroups, and also for the Weyl group $\mathrm{W}(p, q)$. For some special cases explicit forms of the invariant operators are obtained. We also discuss the methods applied, together with problems in some cases, possible further developments and relevance for the supersymmetric theories.
\end{abstract}

\section{Introduction}

One of the main characteristics of a group and the corresponding Lie algebra is the set of invariant operators, i.e. operators which have polynomial structure and are usually called the Casimir operators. Their construction is the first step in finding the representations of the group. In addition, algebraic invariants have immediate significance for constructing invariant equations, derivation of mass formulas and so on.

For the inhomogeneous groups the problem of searching for the Casimir operators and their eigenvalues has been solved in a whole series of works (see, e.g., $[1,2]$ ). Less studied is the question of invariants for the inhomogeneous groups. The Casimir operators for the Poincare group are well known and the Casimir operators for some other inhomogeneous groups have been found: $\operatorname{ISL}(6, C)[3]$ and $\mathrm{IU}(n)$ with different subgroups of translation [4].

Inhomogeneous groups find important application in the gauge theory of gravitation. Kibble [5] generalized the gauge theory for the gravitational field using as the gauge group the (inhomogeneous) Poincare group instead of the (homogeneous) Lorentz group which had been introduced by Utiyama [6]. This allowed, in contrast with Utiyama's theory, to introduce within the framework of only principle of local invariance a set of inhomogeneous quantities (vierbeins and relations among them) in a natural way. There exist analogous theories based on the inhomogeneous version of GL(n) and also the Weyl and the conformal groups. 
Proceeding from the gauge field theories, one can presume that for unified theories inhomogeneous groups in the spaces of higher dimensions can be important (see, e.g., $[7,8]$ ). For the sake of generality, we shall consider all the inhomogeneous groups.

In the case of inhomogeneous groups even the question of existence and the number of invariant operators is rather complicated. We devote this paper to this question in the case of inhomogeneous Lie groups of arbitrary dimension with classical homogeneous subgroups. For some concrete groups of low dimension the explicit forms of the invariant operators are found. For this purpose, we limit our consideration to the most natural inhomogeneous groups, in which translations transform according to the vector representation of the homogeneous subgroup.

As we shall see, from the point of view of invariant operators the cases of homogeneous and inhomogeneous groups differ from each other markedly. We know that for the homogeneous groups all the invariants are polynomial and their number is simply related to the rank of the group. In the case of inhomogeneous groups the invariant operators can be rational (Weyl group), or even not exist at all [IGL $(n, R)$ group], and the number of invariants is not always related to the dimensionality of the group, for instance, $\operatorname{ISL}(n, R)$ group has only one Casimir operator for any $n$.

There exist two methods for finding the invariant operators. We shall use the first method [9-11] for the study of the $\operatorname{IGL}(n, R), \operatorname{ISL}(n, R), \operatorname{ISp}(2 n)$, and $\mathrm{W}(p, q)$ groups. For the study of the $\operatorname{ISO}(p, q), \operatorname{IU}(p, q)$, and $\operatorname{ISU}(p, q)$ groups it will become necessary to use the second method [12-14].

Section 2 describes the essence of the first method.

In Sect. 3 the group $\operatorname{IGL}(n, R)$ of real inhomogeneous general linear transformations is analyzed. It is shown that the Lie algebra of this group has no invariant operator.

Section 4 deals with the study of the group of unimodular real inhomogeneous linear transformations, $\operatorname{ISL}(n, R)$, which is shown to have only one invariant operator for any $n$. The explicit form of the Casimir operator is given for the case of $\operatorname{ISL}(2, R)$.

The group of inhomogeneous pseudo-orthogonal transformations, $\operatorname{ISO}(p, q)$, is considered in Sect. 5, in which also the second method [12-14] is described and used. It is shown that the number of Casimir operators for this group is equal to $\left[\frac{p+q+1}{2}\right]$.

In Sect. 6 the inhomogeneous full unitary group $\mathrm{IU}(p, q)$ and the unimodular (or special) unitary group $\operatorname{ISU}(p, q)$ are studied. It is shown that the invariant operators of these groups are polynomial and their numbers are $(p+q)$ and $(p+q-1)$, respectively.

Section 7 deals with the inhomogeneous symplectic group $\operatorname{ISp}(2 n)$. Its Casimir operators are shown to be polynomial and for their number $\tau$ we have succeeded in obtaining only the inequality $1 \leqq \tau \leqq n$.

In Sect. 8 the Weyl group W $(p, q)$, i.e. the group of motions in pseudoEuclidean space and uniform dilatations in all the coordinate axes, is considered. The invariant operators of this group are shown to be rational and their number is equal to $\left[\frac{p+q-1}{2}\right]$. 


\section{Basic Definitions and the Method}

Let us first recall some definitions and introduce the necessary notation:

(i) The semi-direct product of some group of transformations $G$ and an abelian group (of translations) $T, G \otimes T$, is called the inhomogeneous group.

(ii) The infinite-dimensional associative algebra $A$, which consists of all possible polynomials made out of the generators is called the universal enveloping (or covering) algebra $[14,15]$ of the given Lie algebra. The product in this algebra is the usual associative product of operators. Note that two polynomials are to be considered equal if one can be reduced to the other using the given commutation relations of the Lie algebra.

(iii) Let $S$ be the algebra of usual polynomials of $n$ commuting variables.

(iv) Introduce the adjoint representations of the Lie algebra for the universal enveloping algebra $A$ and for the algebra of polynomials $S$.

In the case of $A$, each generator $F_{j}$ of the Lie algebra acts on an element $u$ of $A$ via the usual Commutator:

$$
\left[F_{j}, u\right] \equiv F_{j} u-u F_{j} .
$$

In the case of $S$, each generator $F_{j}$ acts on a polynomial $p$ from $S$ as a differential operator:

$$
\hat{F}_{j}(p) \equiv \sum_{k, \ell} C_{j \ell}^{k} a_{k} \frac{\partial p}{\partial a_{\ell}},
$$

where the $C_{j \ell}^{k}$ are the structure constants of the group.

(v) The centres $A^{I}$ and $S^{I}$ of the algebras $A$ and $S$ consist of the elements which become zero if any operator from the adjoint representation acts on them.

The set of algebraically independent elements of the centre of the universal enveloping algebra $A^{I}$ is called the set of Casimir operators.

The idea of the method for finding the invariant operators reduces to the following [9-11]. We are given the Lie algebra of the inhomogeneous group. Thus its universal enveloping algebra is defined. The necessity for introducing the universal enveloping algebra can be explained by the fact that the invariant operators which belong to this algebra form its centre. It would be possible to look for the invariant operators of the given Lie algebra by determining the $A^{I}$ directly.

However, it is easier to use an indirect method: together with the universal enveloping algebra, introduce the algebra of usual polynomials by defining its adjoint representation and the centre in a specific way. As it turns out, it is easier to find the $S^{I}$ than the centre of the universal enveloping algebra. The condition that the polynomials belong to the centre of their algebra is formulated, as seen from the definition, in the form of a system of partial differential equations:

$$
\sum_{j, k} C_{i j}^{k} a_{k} \frac{\partial f}{\partial a_{j}}=0,(i=1, \ldots, n) .
$$

Further, one establishes a linear isomorphism between the centre of the algebra of polynomials and the centre of the universal enveloping algebra:

$$
\Phi\left(a_{\alpha_{1}} a_{\alpha_{2}} \ldots a_{\alpha_{r}}\right)=\frac{1}{r !} \sum_{\sigma \in \Pi r} F_{\alpha_{\sigma_{1}}} F_{\alpha_{\sigma_{2}}} \ldots F_{\alpha_{\sigma_{r}}},
$$


where $\prod_{r}$ is the group of permutations of $r$ objects. One can prove [16] that the total number of solutions (polynomial as well as nonpolynomial) of the system (2.3) is given by the formula

$$
\tilde{\tau}=\operatorname{dim} G-r(G),
$$

where $\operatorname{dim} G$ is the dimension of the group; $r(G) \equiv \sup _{\left(a_{1}, \ldots, a_{n}\right)} \operatorname{rank} M$ is the exact upper limit of the rank of the matrix $M$ with respect to the values of the variables $a_{1}, \ldots, a_{n}$, and

$$
M_{i j} \equiv \sum_{k} C_{i j}^{k} a_{k}
$$

From here it follows that the number $\tau$ of polynomial solutions is determined by the inequality

$$
\tau \leqq \operatorname{dim} G-r(G) .
$$

For practical calculations it is convenient to use a criterion [11] which allows us to determine immediately the type of invariants of a given group. According to this criterion, if one can represent any generator of the Lie algebra in the form of a commutator of two others,

$$
[G, G]=G,
$$

then the group has only polynomial invariants. Obviously, for such groups the inequality (2.7) turns out to be an equality. The system (2.3) corresponding to the groups which do not satisfy the condition (2.8) can also have nonpolynomial solutions (rational and even irrational). For such groups one can obtain only the upper bound for the number of polynomial Casimir operators [17].

Thus, the main steps for finding the invariant operators of a given group are the following: 1) one writes down the Lie algebra of the group ; 2) with the help of criterion (2.8) one determines the possible type(s) of invariants; 3 ) one finds the exact upper limit for the rank of the matrix $M$ and obtains the number of invariant operators; 4) for concrete groups one solves the system (2.3) and obtains the explicit form of the invariant operators.

\section{The Group IGL $(n, R)$}

Consider the group of inhomogeneous general real linear transformations $\operatorname{IGL}(n, R)$. Its Lie algebra is given by $[18]$

$$
\begin{gathered}
{\left[I_{\mu v}, I_{\lambda \tau}\right]=\delta_{v \lambda} I_{\mu \tau}-\delta_{\mu \tau} I_{\lambda v},} \\
{\left[I_{\mu v}, P_{\varrho}\right]=-\delta_{\mu \varrho} P_{v},} \\
{\left[P_{\mu}, P_{v}\right]=0 .}
\end{gathered}
$$

We prove that this group has no invariant operators. For this purpose, we show that for some values of the variables $\left(a_{11}, \ldots, a_{n n}, a_{n+1,1}, \ldots, a_{n+1, n}\right)$, the determinant of the matrix $M$, defined in (2.6), is different from zero:

$$
\operatorname{det} M \equiv \operatorname{det} C_{\mu \nu, \varrho \sigma}^{\lambda \tau} a_{\lambda \tau} \neq 0
$$


(for convenience we use double indices). From here it follows that sup rank $M$ $=\operatorname{dim} G$. Hence according to (2.5) there exists no solution for the system $(2.3)$. We present a proof by induction with respect to the dimension of the group.

First we notice that if we write the coordinate transformations in the form

$$
\left(x_{1}^{\prime}, \ldots, x_{n}^{\prime}, 1\right)=\left(x_{1}, \ldots, x_{n}, 1\right)\left(\begin{array}{ccccc}
b_{11} & b_{12} & \ldots & b_{1 n} & 0 \\
\ldots \ldots & \ldots \ldots & \ldots & \ldots \\
b_{n 1} & b_{n 2} & \ldots & b_{n 2} & 0 \\
b_{n+1,1} & b_{n+1,2} & \ldots & b_{n+1, n} & 1
\end{array}\right),
$$

and if we introduce the notation $P_{l} \equiv I_{n+1, l}$, then the generators of the translations can be considered on the same footing as the generators of the homogeneous transformations. In this way the structure constants of the group and the matrix $M$ look like:

$$
\begin{aligned}
C_{\mu v, \varrho \sigma}^{\lambda \tau} & =\delta_{v \varrho} \delta_{\lambda \mu} \delta_{\tau \sigma}-\delta_{\mu \sigma} \delta_{\lambda \varrho} \delta_{\tau v}, \\
M_{\mu v, \varrho \sigma} & =C_{\mu \nu, \varrho \sigma}^{\lambda \tau} a_{\lambda \tau}=\delta_{v \varrho} a_{\mu \sigma}-\delta_{\mu \sigma} a_{\varrho v} .
\end{aligned}
$$

Consider the group $\operatorname{IGL}(1, R)$. Its Lie algebra consists of two generators $I_{11}$ and $P \equiv I_{21}$ and is defined by the relations

$$
\begin{gathered}
{\left[I_{11}, I_{21}\right]=I_{21}} \\
\operatorname{det} M^{(1)}=\left|\begin{array}{cc}
0 & -a_{21} \\
a_{21} & 0
\end{array}\right|=a_{21}^{2} \neq 0 ;
\end{gathered}
$$

i.e., the determinant is indeed different from zero.

Let us illustrate the idea of the proof for the $\operatorname{IGL}(2, R)$ group. Using (3.2), we obtain the determinant of $M^{(2)}$ :

$$
\operatorname{det} M^{(2)}=\left|\begin{array}{cccccc}
0 & a_{12} & -a_{21} & 0 & -a_{31} & 0 \\
-a_{12} & 0 & a_{11}-a_{22} & a_{12} & -a_{32} & 0 \\
a_{21} & -a_{11}+a_{22} & 0 & -a_{21} & 0 & -a_{31} \\
0 & -a_{12} & a_{21} & 0 & 0 & -a_{32} \\
a_{31} & a_{32} & 0 & 0 & 0 & 0 \\
0 & 0 & a_{31} & a_{32} & 0 & 0
\end{array}\right| .
$$

Once can see that: a) this determinant is obtained from the previous determinant $M^{(1)}$ if one adds to the latter, rows and columns with new numbers (12), (22), (31), and (32); b) the rows and the columns (31) and (32) contain only the variables $a_{31}$, $a_{32}$ and only once; c) one of these variables, in this case $a_{32}$, stands in the intersection of the new rows and columns. Therefore if we put $a_{31}=0$, then the determinant of $M^{(2)}$ will be equal, up to a nonzero factor, to the determinant of $M^{(1)}$ :

$$
\operatorname{det} M^{(2)}=a_{32}^{4}\left|\begin{array}{cc}
0 & -a_{21} \\
a_{21} & 0
\end{array}\right|=a_{32}^{4} \operatorname{det} M^{(1)} \neq 0
$$


Let us generalize this result to the case of $\operatorname{IGL}(n, R)$. Suppose that $\operatorname{det} M^{(n-1)} \neq 0$. One can see from (3.2) that in passing from $\operatorname{IGL}(n-1, R)$ to $\operatorname{IGL}(n, R)$ new columns and rows with numbers $(1 n),(2 n), \ldots,(n n)$, $(n+1,1), \ldots,(n+1, n)$ simply appear, but otherwise the structure of the matrix $M$ remains unchanged. In the rows and the columns with numbers $(n+1, k)$ the variables $a_{n+1, l}$ stand, corresponding to the generators of translations and each of such rows and columns contains all the $a_{n+1, l}$ only once. Indeed, from (3.2) it follows that, for instance for the row, one has

$$
M_{n+1 v, \varrho \sigma}=\delta_{v e} a_{n+1, \sigma},
$$

since there is no column with the number $(\varrho, n+1)$, and for any of the $v$ and $\sigma$ $(v, \sigma=1, \ldots, n)$ a corresponding column $(\varrho=v, \sigma)$ exists. One can see from (3.2) that $a_{n+1, n}$ stands in the intersection of the rows and columns for which either $\mu=n+1$, $\sigma=n$ or $\varrho=n+1, v=n$, i.e. in the intersection of those rows and columns which are added in passing from $\operatorname{IGL}(n-1, R)$ to $\operatorname{IGL}(n, R)$. This means

$$
\operatorname{det} M^{(n)} \propto a_{n+1, n}^{(2 n-1)} \operatorname{det} M^{(n-1)} \neq 0 .
$$

Consequently the full linear inhomogeneous real group $\operatorname{IGL}(n, R)$ has no invariants.

\section{The Group $\operatorname{ISL}(n, R)$}

We show that the group of unimodular (or special) real linear inhomogeneous transformations, $\operatorname{ISL}(n, R)$, has only one invariant operator for any $n$.

1) The generators of the homogeneous transformations $H_{i}, I_{\mu v}(i, \mu, v=1, \ldots, n$; $\mu \neq v)$ and translations $P_{\tau}(\tau=1, \ldots, n)$ of the $\operatorname{ISL}(n, R)$ group satisfy the following commutation relations:

$$
\begin{gathered}
{\left[H_{i}, H_{j}\right]=0,} \\
{\left[H_{i}, I_{\mu v}\right]=\delta_{i \mu} I_{i v}-\delta_{i v} I_{\mu i}-\delta_{i+1, \mu} I_{i+1, v}+\delta_{i+1, v} I_{\mu, i+1},} \\
{\left[I_{\mu \nu}, I_{\lambda \tau}\right]=\delta_{\lambda v} I_{\mu \tau}-\delta_{\mu \tau} I_{\lambda v},} \\
{\left[I_{\mu v}, P_{\varrho}\right]=-\delta_{\mu \varrho} P_{v}} \\
{\left[H_{i}, P_{v}\right]=\delta_{i+1, v} P_{i+1}-\delta_{i v} P_{i}} \\
{\left[P_{\mu}, P_{v}\right]=0 .}
\end{gathered}
$$

2) As can be seen, the Lie algebra of the $\operatorname{ISL}(n, R)$ group satisfies the criterion (2.8) which means that its invariants are polynomial.

3) We pass to the determination of the number of Casimir operators. The number of parameters of the $\operatorname{ISL}(n, R)$ group is $n^{2}-1+n=n(n+1)-1$ which is odd. The antisymmetry of the structure constants of the group entails that the matrix $M$ is also antisymmetric. But the determinant of an antisymmetric matrix of odd order is always equal to zero. This means that this group has at least one Casimir operator. 
Let us show that there is exactly one invariant. For this purpose, according to (2.7), it is necessary to prove that the determinant of the matrix $M$, defined in (2.6), has at least one nonzero minor of order $(\operatorname{dim} G-1)$.

In order to write the matrix elements in a similar way, we use the fact that the generator of the Cartan subgroup of $\operatorname{SL}(n, R)$ can be expressed in terms of the generators of $\operatorname{GL}(n, R): H_{i}=I_{i i}-I_{i+1, i+1}$. Then for the matrix $M$ we obtain

$$
M_{\mu v, \varrho \sigma}=C_{\mu v, \varrho \sigma}^{\lambda \tau} a_{\lambda \tau}=\delta_{v \varrho} a_{\mu \sigma}-\delta_{\mu \sigma} a_{\varrho v},(\mu, \varrho=1, \ldots, n+1 ; v, \sigma=1, \ldots, n) .
$$

In order to make the form of the matrix simple (in analogy with Sect. 3), we put equal to zero all the variables $a_{\lambda \tau}$ with the exception of those which correspond to the Cartan subalgebra and to the subalgebra of translations $a_{n+1, k} \equiv p_{k}$. In the determinant thus obtained we choose the minor of order $(\operatorname{dim} G-1)$ by crossing out the row and the column with the number $(n+1,1)$. This minor is as follows:

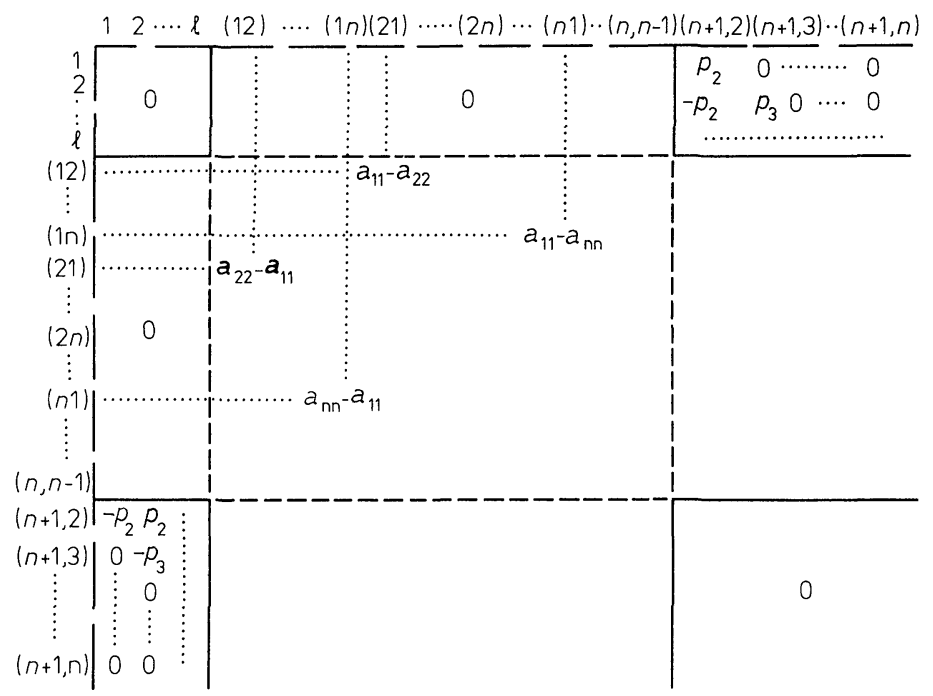

The elements of the minors in the upper left and the lower right corners are zero because of commutativity of both the generators $H_{j}$ and $P_{\mu}$.

In the rows and columns of the middle minor, surrounded by dashed lines, there is only one element each, since the generator $\left(I_{i i}-I_{i+1, i+1}\right)$ is obtained only in the commutation of $\left[I_{i, i+1}, I_{i+1, i}\right]$. This means that this minor is different from zero. The elements of the minors which are disposed on the left and above this latter minor are equal to zero because we have put equal to zero all the variables $a_{\lambda \tau}$ which they consist of. The elements of the two remaining minors are determined by the relation: $\left[H_{i}, P_{j}\right]=\delta_{j, i+1} P_{j}-\delta_{i j} P_{j}$. Expansion of the minor $M$ with respect to the first row results in a minor which contains as before one element in the first row. By continuing this procedure, we arrive at the minor in the middle, which we have shown to be nonzero.

Thus the whole minor $M$ is indeed different from zero and consequently the group $\operatorname{ISL}(n, R)$ has exactly one Casimir operator.

4) As an illustration we compute the Casimir operator of the $\operatorname{ISL}(2, R)$ group. The Lie algebra of this group consists of the operators $H_{1} \equiv\left(I_{11}-I_{22}\right), I_{12}, I_{21}$, 
$P_{1} \equiv I_{31}$, and $P_{2} \equiv I_{32}$. We denote their corresponding variables in the algebra of polynomials by $a_{1}, \ldots, a_{5}$. According to $(2.3)$ we obtain the following system of equations :

$$
\begin{gathered}
2 a_{2} \frac{\partial f}{\partial a_{2}}-2 a_{3} \frac{\partial f}{\partial a_{3}}-a_{4} \frac{\partial f}{\partial a_{4}}+a_{5} \frac{\partial f}{\partial a_{5}}=0, \\
-2 a_{2} \frac{\partial f}{\partial a_{1}}+a_{1} \frac{\partial f}{\partial a_{3}}-a_{5} \frac{\partial f}{\partial a_{4}}=0, \\
2 a_{3} \frac{\partial f}{\partial a_{1}}-a_{1} \frac{\partial f}{\partial a_{2}}-a_{4} \frac{\partial f}{\partial a_{5}}=0, \\
a_{4} \frac{\partial f}{\partial a_{1}}+a_{5} \frac{\partial f}{\partial a_{2}}=0 \\
-a_{5} \frac{\partial f}{\partial a_{1}}+a_{4} \frac{\partial f}{\partial a_{3}}=0 .
\end{gathered}
$$

In order to solve this system, we express all the partial derivatives in terms of $\frac{\partial f}{\partial a_{1}}$ and $\frac{\partial f}{\partial a_{4}}$. Equations (4.3), (4.4), and (4.5) give

$$
\begin{aligned}
& \frac{\partial f}{\partial a_{2}}=-\frac{a_{4}}{a_{5}} \frac{\partial f}{\partial a_{1}}, \\
& \frac{\partial f}{\partial a_{3}}=\frac{a_{5}}{a_{4}} \frac{\partial f}{\partial a_{1}}, \\
& \frac{\partial f}{\partial a_{5}}=\left(\frac{2 a_{3}}{a_{4}}+\frac{a_{1}}{a_{5}}\right) \frac{\partial f}{\partial a_{1}} .
\end{aligned}
$$

By substituting these expressions into (4.1) and (4.2) we obtain two equivalent equations. Let us consider, for instance, Eq. (4.1) which gives

$$
\left(a_{1}-2 a_{2} \frac{a_{4}}{a_{5}}\right) \frac{\partial f}{\partial a_{1}}-a_{4} \frac{\partial f}{\partial a_{4}}=0 .
$$

The solution of this question is the function

$$
f=f\left(g, a_{2}, a_{3}, a_{5}\right), \quad g=\frac{a_{2} a_{4}^{2}}{a_{5}}-a_{1} a_{4} .
$$

By differentiating the $f$ as a compound function and using the expressions (4.6), we obtain the form of all partial derivatives up to a factor $h=h\left(a_{2}, a_{3}, a_{5}\right)$. For its determination, we express the partial derivatives in the system of Eqs. (4.1)-(4.5) in terms of $\frac{\partial f}{\partial a_{1}}$ and $\frac{\partial f}{\partial a_{5}}$, and again we find expressions for all the partial derivatives which contain another factor. Comparing both expressions for the partial derivatives we find $h=h\left(a_{1}, a_{3}, a_{5}\right)$. After integrating the expressions for the partial derivatives and comparing them with each other, we obtain the solution for the system of Eqs. (4.1)-(4.5) in the form $f=a_{1} a_{4} a_{5}+a_{3} a_{5}^{2}-a_{2} a_{4}^{2}$. Therefore, in 
accordance with (2.4) we obtain for the Casimir operator of the $\operatorname{ISL}(2, R)$ group the expression

$$
C=\left(I_{11}-I_{22}\right) P_{1} P_{2}+I_{21} P_{2}^{2}-I_{12} P_{1}^{2},
$$

where for passing to a more compact form, as compared with the symmetric form, we have used the commutation relations.

\section{The Group $\operatorname{ISO}(p, q)$}

Consider the group of inhomogeneous pseudo-orthogonal transformations $\operatorname{ISO}(p, q)$.

1) Its Lie algebra has the form

$$
\begin{aligned}
{\left[I_{\mu v}, I_{\varrho \sigma}\right] } & =g_{v \varrho} I_{\mu \sigma}+g_{\mu \sigma} I_{v \varrho}-g_{\mu \varrho} I_{v \sigma}-g_{v \sigma} I_{\mu \varrho}, \\
{\left[I_{\mu \nu}, P_{\varrho}\right] } & =g_{v \varrho} P_{\mu}-g_{\mu \varrho} P_{v}, \\
{\left[P_{\mu}, P_{v}\right] } & =0, \\
g & =\operatorname{diag}(\underbrace{-1,-1, \ldots,-1}_{p}, \underbrace{1, \ldots, 1}_{q}) .
\end{aligned}
$$

2) From (5.1), according to the criterion (2.8), it follows that the group $\operatorname{ISO}(p, q)$ can only have invariant operators of polynomial form.

3) We now come to the determination of the number of Casimir operators. First we determine the upper limit using the method described in Sect. 2. The elements of the matrix $M$ defined in Sect. 2 for the group $\operatorname{ISO}(p, q)$ have the form

$$
\begin{aligned}
M_{\mu v, \varrho \sigma} & =C_{\mu \nu, \varrho \sigma}^{\lambda \tau} a_{\lambda \tau} \\
& =\left(1-\delta_{v, n+1} \delta_{\sigma, n+1}\right)\left(g_{v \varrho} a_{\mu \sigma}+g_{\mu \sigma} a_{v \varrho}-g_{\mu \varrho} a_{v \sigma}-g_{v \sigma} a_{\mu \varrho}\right) .
\end{aligned}
$$

In (5.2) we put all the variables $a_{\lambda \tau}$ equal to zero, except for the variables corresponding to translations and to the generators $I_{2 i, 2 i-1}\left(i=1,2, \ldots,\left[\frac{n}{2}\right]\right)$. Then the matrix can be written in the following form (for definiteness consider the case of even $n$; the analysis for odd $n$ will be analogous):

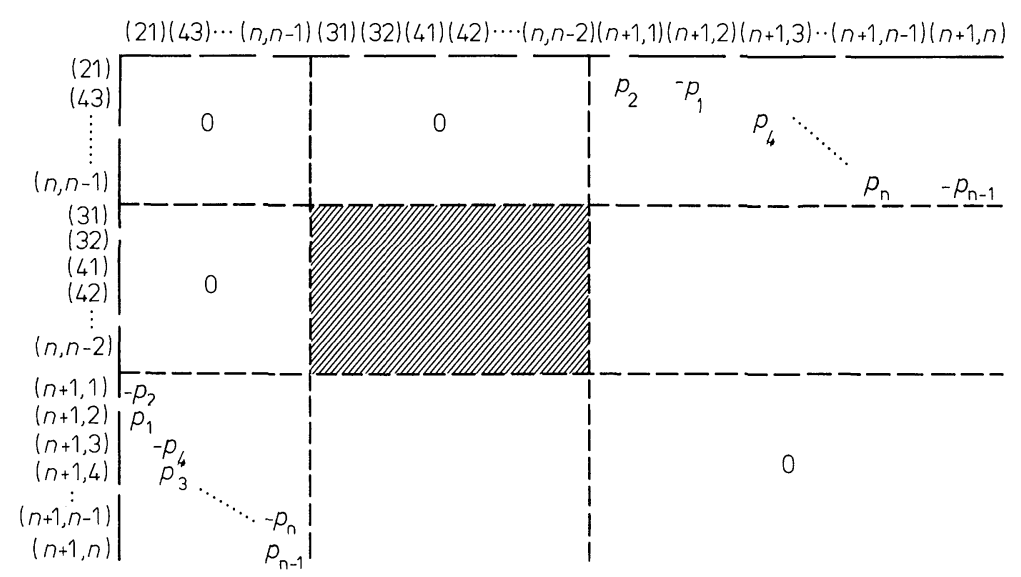


The upper left and the lower right minors have zero elements because of commutativity of the corresponding generators. The minors on the left and above the shaded area are zero because of our choice of values for the variables $a_{\lambda \tau}$. We cross out the rows and columns with the numbers $(n+1,2 k), k=1, \ldots, \frac{n}{2}=\left[\frac{n+1}{2}\right]$ (since $n$ is even) and we show that the leftover minor $M^{\prime}$ is different from zero. The expansion of $M^{\prime}$ with respect to the rows and columns which correspond to translations gives $M^{\prime} \propto p_{2}^{2} p_{4}^{2} \ldots p_{n}^{2} M_{n}^{\prime \prime}$, where $M_{n}^{\prime \prime}$ is the shaded minor in the middle.

We prove by induction that $M_{n}^{\prime \prime} \neq 0$. For $n=4$ one has

$$
M_{4}^{\prime \prime}=\left|\begin{array}{cccc}
0 & a_{21} & a_{43} & 0 \\
-a_{21} & 0 & 0 & a_{43} \\
-a_{43} & 0 & 0 & a_{21} \\
0 & -a_{43} & -a_{21} & 0
\end{array}\right|=a_{43}^{4}+a_{21}^{4}-2 a_{43}^{2} a_{21}^{2} \neq 0 .
$$

Let $M_{n-2}^{\prime \prime} \neq 0$ for $n-2$. We show that this is also true for $n$. The minor $M_{n}^{\prime \prime}$ has the form

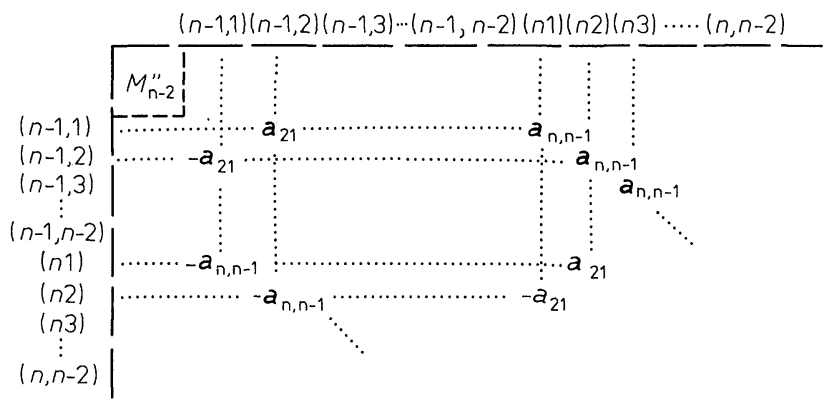

Let us put the variable $a_{n, n-1}=0$ (one can do this since this element does not enter into the minor $M_{n-2}^{\prime \prime}$ ). Then in each row and column which has been added, only one element is left. The expansion of the minor in terms of these rows and columns brings us to the minor $M_{n-2}^{\prime \prime}$, which is different from zero. Thus the upper limit $r(G)$ for the rank of the matrix $M$ is determined by the inequality

$$
r(G) \geqq \operatorname{dim} G-\left[\frac{n+1}{2}\right] .
$$

From here, according to (2.7), we obtain for the number of Casimir operators of the group $\operatorname{ISO}(p, q)$ the inequality

$$
\tau \leqq\left[\frac{n+1}{2}\right] .
$$

In order to obtain the exact value for the number of Casimir operators, we utilize still another method [12-14] which allows us to find the lower limit for the number of invariant operators $\tau$. In this method the problem of finding the Casimir operators of an inhomogeneous group is reduced to the use of the Casimir operators of the homogeneous group. This can be done if we "expand" the 
inhomogeneous group to the corresponding homogeneous one of higher dimensions. The procedure of expanding the group $\operatorname{ISO}(p, q)$ under consideration amounts to the following.

Denote

$$
J_{\mu}=\frac{1}{2}\left(P^{v} M_{\mu v}+M_{\mu \nu} P^{v}\right)
$$

An obvious invariant of $\operatorname{ISO}(p, q)$ is the operator $P^{2}=g_{\mu \nu} P^{\mu} P^{v}$, where $g=\operatorname{diag}(\underbrace{-1,-1, \ldots,-1}_{p}, \underbrace{1, \ldots, 1}_{q})$ is the metric tensor.

Then, as can be checked, the operator

$$
M_{\mu, n+1}=\left(P^{2}\right)^{-1 / 2} J_{\mu}
$$

together with the generators of the homogeneous subgroup of the $\operatorname{ISO}(p, q)$ group constitute the algebra of $\mathrm{SO}(p, q+1)$, which is the expansion of $\operatorname{ISO}(p, q)$.

By using (5.4) and (5.5) one can prove the theorem [12]: If an element of the enveloping algebra of $\operatorname{ISO}(p, q)$ commutes with all the $M_{\mu \nu}$ and $J_{\mu}$, then it commutes also with all $P_{\mu}$.

If we substitute now (5.4) and (5.5) into the expression for the invariant of the homogeneous group of $\mathrm{SO}(p, q+1)$ and multiply it by a sufficiently high power of $P^{2}$, then we obtain a polynomial operator which commutes with all $M_{\mu \nu}$ and $J_{\mu}$, which means also with all $P_{\mu}$, i.e. one obtains the invariant operator of the inhomogeneous group.

From the definition of the expansion, it is seen that the enveloping algebras of the groups $\operatorname{ISO}(p, q)$ and $\mathrm{SO}(p, q+1)$, in general, do not coincide. From the construction it follows that the Casimir operators of the group $\mathrm{SO}(p, q+1)$ also belong to the centre of the universal enveloping algebra of the initial Lie algebra. The converse, in general, is not true: the Casimir operator of the group $\operatorname{ISO}(p, q)$ may not belong to the enveloping algebra of the group $\mathrm{SO}(p, q+1)$. Thus, this method gives for the number of Casimir operators only the lower limit

$$
\tau_{\mathrm{ISO}(p, q)} \geqq \tau_{S \mathrm{O}(p, q+1)}=\left[\frac{n+1}{2}\right], \quad n=p+q .
$$

The comparison of (5.3) and (5.6) gives the exact number of Casimir operators for the inhomogeneous pseudo-orthogonal group $\operatorname{ISO}(p, q)$ :

$$
\tau=\left[\frac{p+q+1}{2}\right] \text {. }
$$

Notice that the method described is not suitable for all the groups, since one cannot always succeed in constructing the necessary expansion without increasing the dimension of the subgroup of translations (which takes place, for instance, in the case of linear and the symplectic groups). The explicit form of the Casimir operators is determined by the system of Eqs. (2.3). By solving it one can obtain in accordance with (5.7), that the group $\operatorname{ISO}(1,1)$, for example, has one Casimir operator $C=P_{1}^{2}-P_{2}^{2}$, and the group ISO(3) has $C_{1}=P_{1}^{2}+P_{2}^{2}+P_{3}^{2}$ and $C_{2}=L_{1} P_{1}+L_{2} P_{2}+L_{3} P_{3}$, where $L_{i}$ are the generators of the subgroup of ro- 
tations. The method of expansion also leads to the same form of invariant operators.

\section{The Groups $\operatorname{IU}(p, q)$ and $\operatorname{ISU}(p, q)$}

In this section we investigate the inhomogeneous full unitary group $\mathrm{IU}(p, q)$ and the inhomogeneous special unitary group $\operatorname{ISU}(p, q)$.

1) The Lie algebra of $\mathrm{IU}(p, q)$ is given by

$$
\begin{gathered}
{\left[L_{\mu \nu}, L_{\lambda \sigma}\right]=g_{v \lambda} L_{\mu \sigma}-g_{\lambda \mu} L_{v \sigma}+g_{v \sigma} L_{\lambda \mu}-g_{\sigma \mu} L_{\lambda v},} \\
{\left[L_{\mu \nu}, Q_{\lambda \sigma}\right]=g_{v \lambda} Q_{\mu \sigma}-g_{\lambda \mu} Q_{v \sigma}+g_{v \sigma} Q_{\lambda \mu}-g_{\sigma \mu} Q_{\lambda \nu},} \\
{\left[Q_{\mu v}, Q_{\lambda \sigma}\right]=-g_{v \lambda} L_{\mu \sigma}-g_{\lambda \mu} L_{v \sigma}+g_{v \sigma} L_{\lambda \mu}+g_{\sigma \mu} L_{\lambda \nu},} \\
{\left[L_{\mu v}, P_{\lambda}\right]=g_{v \lambda} P_{\mu}-g_{\lambda \mu} P_{v}, \quad\left[Q_{\mu v}, R_{\lambda}\right]=-g_{v \mu} P_{\mu}-g_{\lambda \mu} P_{v},} \\
{\left[Q_{\mu \nu}, P_{\lambda}\right]=g_{v \lambda} R_{\mu}+g_{\lambda \mu} R_{v}, \quad\left[L_{\mu v}, R_{\lambda}\right]=g_{v \lambda} R_{\mu}-g_{\lambda \mu} R_{v},} \\
L_{\mu \nu}=-L_{v \mu}, \quad(\mu, v, \lambda, \sigma=1, \ldots, n) . \\
Q_{\mu \nu}=+Q_{v \mu},
\end{gathered}
$$

In the case of $\operatorname{ISU}(p, q)$ it is sufficient to consider $\left(Q_{\mu \mu}-Q_{\mu+1, \mu+1}\right)$ as the elements of the basis, instead of $Q_{\mu \mu}$.

2) From the Lie algebra one can see that $\operatorname{ISU}(p, q)$ satisfies the criterion (2.8): $[\operatorname{ISU}(p, q), \operatorname{ISU}(p, q)]=\operatorname{ISU}(p, q)$, but the Lie algebra of $\operatorname{IU}(p, q)$ does not satisfy this criterion. This means that the inhomogeneous unimodular unitary group has only polynomial invariants.

3) We come to the determination of the number of invariant operators. Let us first consider the group IU $(p, q)$. The upper limit for the number of invariant operators is determined by the rank of the matrix $M$, defined in (2.6), which in our case has the following form (all the variables except the ones corresponding to $Q_{\mu \mu}$, $P_{\mu}$, and $R_{\mu}$ have been put equal to zero):

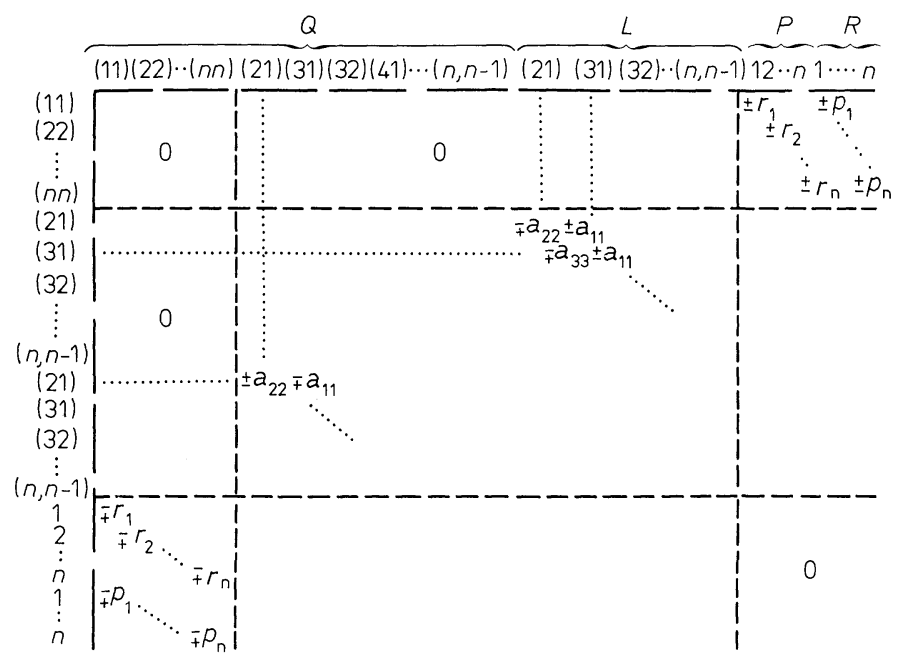


By crossings out the rows and the columns corresponding to $R$-translations, one can see that the leftover minor of order $(\operatorname{dim} G-n)$ (where $\operatorname{dim} G$ is the dimension of the group) is different from zero since in each row and column, there is only one element. This means that, according to (2.7), the number of invariant operators of the group $\mathrm{IU}(p, q)$ satisfies the inequality

$$
\tau_{\mathrm{IU}(p, q)} \leqq n,(n=p+q) .
$$

For the determination of the lower limit for the number of invariants we use the method of expansion [12-14]. In order to expand the considered inhomogeneous group up to $\mathrm{U}(p, q+1)$ we introduce the following operators [taking into account the fact that $P^{2}+R^{2}=P_{\mu} P^{\mu}+R_{\mu} R^{\mu}$ is the invariant of the $\mathrm{IU}(p, q)$ group]:

$$
\begin{aligned}
& L_{\mu, n+1}=\frac{1}{2}\left(P^{2}+R^{2}\right)^{-1 / 2}\left(P^{v} L_{\mu \nu}+L_{\mu \nu} P^{\nu}-R^{v} Q_{\mu \nu}-Q_{\mu \nu} R^{v}\right), \\
& Q_{\mu, n+1}=\frac{1}{2}\left(P^{2}+R^{2}\right)^{-1 / 2}\left(R^{v} L_{\mu \nu}+L_{\mu \nu} R^{\nu}-P^{v} Q_{\mu \nu}+Q_{\mu \nu} P^{v}\right), \\
& Q_{n+1, n+1}=\frac{1}{2}\left(P^{2}+R^{2}\right)^{-1 / 2}\left[P^{\mu} R^{v} L_{\mu \nu}+\left(P^{\mu} P^{\nu}+R^{\mu} R^{v}\right) Q_{\mu \nu}\right] .
\end{aligned}
$$

These operators together with the generators of the homogeneous subalgebra of the group $\mathrm{IU}(p, q)$ constitute, as can be checked, the Lie algebra of $\mathrm{U}(p, q+1)$. Furthermore, in analogy to the case of the orthogonal group, one can separate out of the known Casimir operators of $U(p, q+1)$ the invariants of the $\mathrm{IU}(p, q)$. At the same time, it is obvious that all the invariant operators obtained by this method are polynomial [ we can multiply, in case of necessity, by an arbitrary power of $\left.\left(P^{2}+R^{2}\right)\right]$.

However, in contrast to the pseudo-orthogonal inhomogeneous group, the number of generators of $\operatorname{IU}(p, q)$, which is equal to $n^{2}+2 n$, is one less than the dimension of $U(p, q+1)$. Therefore, in reality, not all the generators of expansion of $\mathrm{IU}(p, q)$ are independent, which dictates some relation among the $(n+1)$ Casimir operators of the expanded group of $\operatorname{IU}(p, q)$.

Thus, by using the second method, we obtain the lower limit for the number of invariant operators :

$$
\tau \geqq n
$$

Comparison of (6.1) and (6.2) gives the exact number of Casimir operators of the $\mathrm{IU}(p, q)$ group: $\tau_{\mathrm{IU}(p, q)}=p+q$. Notice that, although $\mathrm{IU}(p, q)$ does not satisfy the criterion (2.8), all its invariants are polynomial.

Consider now the unimodular unitary group $\operatorname{ISU}(p, q)$. The process of expansion for this group has not been studied, but one can easily perform it on the basis of the $\mathrm{IU}(p, q)$ group. For this purpose it is enough to consider, in the initial and in the expanded groups, not the $Q_{\mu \mu}$ as the basic elements of the algebra but the $\left(Q_{\mu \mu}-Q_{\mu+1, \mu+1}\right)$ (the other elements remain unchanged). Since the invariant of first order for the group $\mathrm{SU}(p, q+1)$ is identically zero, we obtain [analogously to $\mathrm{IU}(p, q)]$ :

$$
\tau_{\mathrm{ISU}(p, q)} \geqq n-1 .
$$

Let us show that (6.3) is in fact an equality. Crossing out in the usual way the rows and columns corresponding to $R_{2}, \ldots, R_{n}$, and leaving nonzero the variables 
corresponding to $\left(Q_{\mu \mu}-Q_{\mu+1, \mu+1}\right), R_{v}, P_{1}$, i.e. $a_{\mu}, r_{v}, p_{1}$, up to a nonzero factor we arrive at the minor of order $(\operatorname{dim} G-n+1)$ :

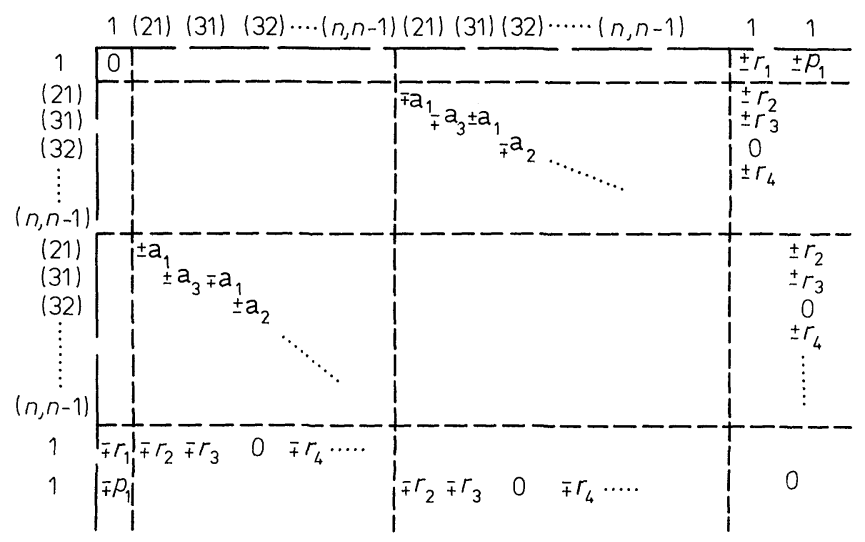

Putting now $a_{1}=0$ and expanding in terms of $Q_{21}, L_{21}$ rows and columns, we obtain a minor in each row and column of which only one element remains nonzero. Thus, the number of Casimir operators of the $\operatorname{ISU}(p, q)$ is equal to $\tau_{\mathrm{ISU}(p, q)}=p+q-1$.

As an example we give expressions for the invariants of the IU(2) group. Solving the system of partial differential equations (2.3), in analogy to the case of the $\operatorname{ISL}(2, R)$ group (Sect. 4$)$, we obtain

$$
\begin{gathered}
C_{1}=P^{2}+R^{2} \\
C_{2}=\left(P_{2}^{2}+R_{2}^{2}\right) Q_{11}+\left(P_{1}^{2}+R_{1}^{2}\right) Q_{22}+2\left(P_{1} R_{2}-P_{2} R_{1}\right) L_{21} \\
-2\left(P_{1} P_{2}+R_{1} R_{2}\right) Q_{21} .
\end{gathered}
$$

One can bring to the same form the invariants of IU(2) obtained in Ref. 12.

\section{The Inhomogeneous Symplectic Group ISp(2n)}

1) The Lie algebra of $\operatorname{ISp}(2 n)$ is given by

$$
\begin{aligned}
& {\left[I_{\mu v}, I_{\varrho \lambda}\right]=\sigma_{v \varrho} I_{\mu \lambda}+\sigma_{v \lambda} I_{\mu \varrho}+\sigma_{\mu \varrho} I_{v \lambda}+\sigma_{\mu \lambda} I_{v \varrho},} \\
& {\left[I_{\mu \nu}, P_{\varrho}\right]=\sigma_{v \varrho} P_{\mu}+\sigma_{\mu \varrho} P_{v} \text {, }} \\
& \sigma=\left(\begin{array}{rr:ll} 
& & 1 & \\
0 & & \ddots & \\
& & & \\
\hdashline 1 & & & \\
\hdashline & & 0
\end{array}\right), \mu, v, \varrho, \lambda= \pm 1, \pm 2, \ldots, \pm n .
\end{aligned}
$$


2) One can see from (7.1) that the Lie algebra of the $\operatorname{ISp}(2 n)$ group satisfies the criterion (2.8) and therefore has only polynomial invariants.

3) For the number of Casimir operators of the symplectic group we have succeeded in obtaining only an interval for its value. For the symplectic group the method of expansion is not suitable since it increases the dimension of the subgroup of translations. Therefore, using the method presented in Sect. 2 and Ref. 11 we shall obtain the upper limit for the number of Casimir operators:

$$
\tau_{\mathrm{ISp}(2 n)} \leqq n
$$

For this purpose, following the usual procedure of Sects. 2-6, we write down the matrix $M$, defined by (2.6), leaving nonzero the variables $a_{\lambda \tau}$ corresponding to the Cartan subalgebra $\left(I_{i,-i}, i=1, \ldots, n\right)$ and to the translations:

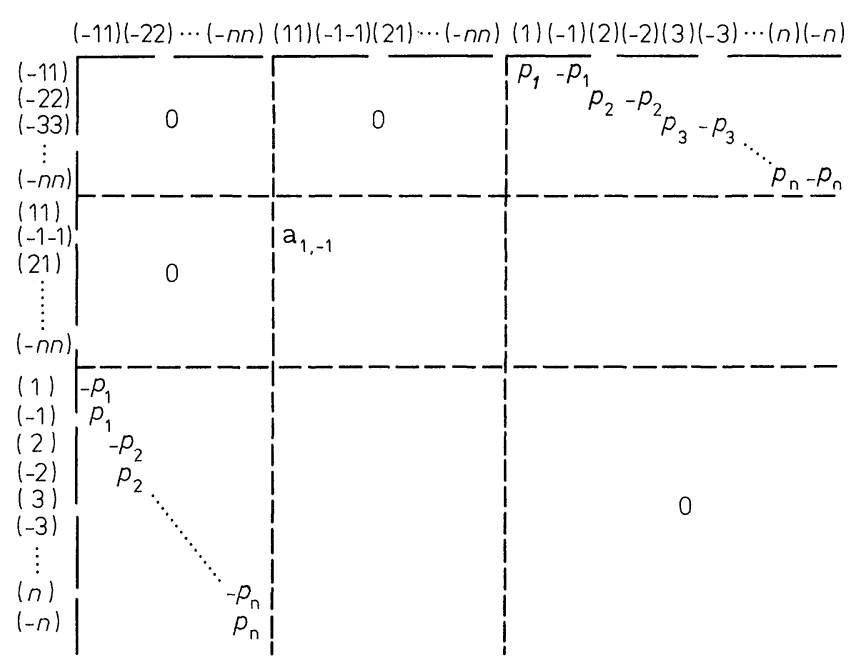

Crossing out $n$ rows and columns, corresponding to the translations with negative indices we come to the nonzero minor since it has only one element in each row and column. From this the formula (7.2) follows.

We can show that there exists at least one Casimir operator for the group $\operatorname{ISp}(2 n)$, i.e. there exists a lower nonzero limit for the number of Casimir operators, $\tau$. For this purpose let us first consider the group ISp(2). It is locally isomorphic to $\operatorname{ISL}(2, R)$. Therefore, using the results of Sect. 4, we obtain the Casimir operator of ISp(2) group:

$$
C_{2}=\sum_{\alpha, \beta, \gamma, \varrho=1}^{-2} P_{\alpha} \sigma_{\alpha \beta} I_{\beta \gamma} \sigma_{\gamma \varrho} P_{\varrho} .
$$

But a similar operator is the invariant of the $\operatorname{ISp}(2 n)$ group for any $n$. Indeed,

$$
\begin{aligned}
{\left[P_{\alpha} \sigma_{\alpha \beta} I_{\beta \gamma} \sigma_{\gamma \varrho} P_{\varrho}, P_{\tau}\right] } & =P_{\alpha} \sigma_{\alpha \beta}\left(\sigma_{\beta \tau} P_{\gamma}+\sigma_{\gamma \tau} P_{\beta}\right) \sigma_{\gamma \varrho} P_{\varrho} \\
& =P_{\alpha} \sigma_{\alpha \beta} P_{\beta} P_{\tau}-P_{\tau} P_{\gamma} \sigma_{\gamma \varrho} P_{\varrho}=0,
\end{aligned}
$$

assuming summation with respect to repeated indices. 
Analogously one can show that the generators $I_{\mu \nu}$ also commute with the operators $C_{2 n}$, i.e. the $C_{2 n}$ are the Casimir operators of the $\operatorname{ISp}(2 n)$ group.

Let us mention that searching for other Casimir operators of the $\operatorname{ISp}(2 n)$ group with the help of tensor analysis is difficult. All contractions with antisymmetric tensors give zero because of $I_{\mu \nu}=I_{\nu \mu}$; symmetric tensors do not give contractions which commute with $P_{\tau}$. Therefore operators of type (7.3) with a different number of $I_{\mu \nu}$ are left. However, operators of higher order with an odd number of $I_{\mu \nu}$ do not commute with $P_{\tau}$, and operators with an even number are reduced to lower orders. For instance, by placing the generators in opposite order in the operator

$$
C_{2 n}^{\prime}=P_{\alpha} \sigma_{\alpha \beta} I_{\beta \gamma} \sigma_{\gamma \varrho} I_{\varrho \tau} \sigma_{\tau \nu} P_{v},
$$

we obtain the sum of commutators $(6 n+2) C_{2 n}$. Then, using the fact that the quadratic form $\sigma$ is antisymmetric, we find $C_{2 n}^{\prime}=(3 n+1) C_{2 n}$.

Thus for the number of Casimir operators $\tau$ for the $\operatorname{ISp}(2 n)$ group we obtain the inequalities $1 \leqq \tau \leqq n$.

\section{Invariant Operators of the (Inhomogeneous) Weyl Group W( $(p, q)$}

In Sects. 3-7 we have considered questions concerning invariant operators of inhomogeneous groups with classical homogeneous subgroups. Here we study the analogous problem for the Weyl group $\mathrm{W}(p, q)$ of arbitrary dimension.

The Weyl group is the group of motions in pseudo-Euclidean space and uniform expansion with respect to all the coordinate axes:

$$
\mathrm{W}(p, q) \equiv(\mathrm{SO}(p, q) \otimes D) \otimes T,
$$

where the sign $\otimes$ denotes the semi-direct product.

1) From the structure of this group (direct product) it is obvious that dilatations commute with rotations. Therefore, compared with the inhomogeneous pseudo-orthogonal group $\operatorname{ISO}(p, q)$ of Sect. 5, only the commutation relations of $P_{v}$ with the generator of dilatations $D$ are new:

$$
\left[D, P_{v}\right]=-P_{v} .
$$

2) The Lie algebra of $\mathrm{W}(p, q)$ does not satisfy any of the sufficiency conditions for the existence of only polynomial operators (Sect. 2). In addition, as we shall see below, $\mathrm{W}(p, q)$ is an example of a group which has only rational invariants. Therefore, we consider briefly the definition of rational invariants of Lie algebra [11].

Let us introduce the following concepts:

(a) The universal enveloping (or covering) algebra $A$ of a Lie algebra is an algebra without zero-divisors [20], i.e. from the condition $u v=0,(u, v \in A)$, it follows that either $u=0$ or $v=0$. Therefore one can define the quotient field $D(A)$, i.e. a field whose elements have the form $u v^{-1},(u, v \in A, v \neq 0)$. Commutators in the quotient field can be calculated using the formula

$$
\left[u, v^{-1}\right] \equiv u v^{-1}-v^{-1} u=-v^{-1}[u, v] v^{-1} .
$$


(b) Elements of the quotient field $D(A)$ which commute with all the generators of the group are called rational invariants.

(c) An Element $g$ of the universal enveloping algebra $A$ is called a relative invariant of the Lie algebra of the group $G$, if

$$
\left[F_{j}, g\right]=\lambda_{j} g \quad(j=1, \ldots, \operatorname{dim} G)
$$

where

$F_{j}$ is a generator of the Lie algebra of $G$,

$\operatorname{dim} G$ is the $\operatorname{dimension}$ of the group, and

$\lambda_{j}$ is a numerical factor.

Let us show that $u$ and $v$ being relative invariants with equal $\lambda_{j}$ is the necessary condition for the invariance of $u v^{-1}$.

Indeed, using (8.2) we find

$$
\left[F_{j}, u v^{-1}\right]=\left[F_{j}, u\right] v^{-1}-u v^{-1}\left[F_{j}, v\right] v^{-1}=\lambda_{j} u v^{-1}-u v^{-1} \lambda_{j} v v^{-1}=0 .
$$

Conversely, from the invariance requirement for $u v^{-1}$ we get $\left[F_{j}, u\right]\left[F_{j}, v\right]^{-1}$ $=u v^{-1}$.

$D(A)$ is algebraically generated by elements of the form $u v^{-1}$, where $u$ and $v$ are relative invariants with equal $\lambda_{j}[11]$. In order to find the number of invariant operators we can use the method described in Sect. 2 and Ref. 11, with the difference that in the present case one can establish an isomorphism between the centre of the quotient field $D(A)$ and the centre of the algebra of rational functions. However, in the case of the Weyl group another method turns out to be more convenient. This method is based on the fact that the problem of finding the number of invariants of the $\mathrm{W}(p, q)$ group can be reduced to the problem of obtaining the number of Casimir operators for its pseudo-orthogonal inhomogeneous subgroup $\operatorname{ISO}(p, q)$. In fact, according to what has been said, instead of finding the absolute invariants, we can look for relative ones. As we shall show the relative invariants of the Weyl group do not include the operators of dilatation and are the absolute invariants of $\operatorname{ISO}(p, q)$ group. Consequently invariant operators of the Weyl group are presented in the form of the ratio of Casimir operators of the inhomogeneous pseudo-orthogonal subgroup.

3) Let us now find the number of invariants. The Lie algebra of ISO $(p, q)$ can not have relative invariants since it is a derived Lie algebra [20], i.e. $\operatorname{ISO}(p, q)$ $=[\mathrm{W}(p, q), \mathrm{W}(p, q)]$. Therefore, in order to prove that the generator of dilatation does not enter into the relative invariants, it is sufficient to show that the following commutation relations are impossible:

$$
\begin{aligned}
{\left[P_{\mu}, C_{D}\right] } & =0, \\
{\left[M_{\mu \nu}, C_{D}\right] } & =0,
\end{aligned}
$$

where the element $C_{D}$ of the universal enveloping algebra $A_{W}$ contains $D$.

Operator $C_{D}$ can be written in the form

$$
C_{D}=\sum_{n=0}^{N} C_{n} D^{n},
$$


where $C_{n} \in A_{W}$ and does not contain the operator $D$. From (8.4) it follows that $\left[M_{\mu \nu}, C_{n}\right]=0$ for any $n$. In other words, $C_{n}$ is an invariant operator of the homogeneous pseudo-orthogonal group. The other relation of (8.4) gives

$$
\sum_{n=0}^{N}\left(\left[P_{\mu}, C_{n}\right] D^{n}+C_{n}\left[P_{\mu}, D^{n}\right]\right)=0 .
$$

Using (8.1) and putting equal to zero the coefficients of different powers of $D$, we obtain

$$
\left[P_{\mu}, C_{n}\right]=\sum_{k=1}^{N-n} a_{k} C_{n+k} P_{\mu}
$$

where $a_{k}$ are some numerical coefficients.

The basis of invariants of the homogeneous subgroup consists of monomials which are contracted in all the indices (this follows from the Gel'fand theorem [9]). Let us introduce the notations $\prod_{\mu \nu}^{n} \equiv I_{\mu \sigma_{1}} I_{\sigma_{1} \sigma_{2}} \ldots I_{\sigma_{n} v}$ and $\prod^{n} \equiv \prod_{\mu \mu}^{n}$. Then an arbitrary invariant is the sum of terms of the form

$$
\prod^{n_{1}} \prod^{n_{2}} \ldots \prod^{n_{k}} \prod_{\mu_{1} v_{1}}^{m_{1}} \ldots \prod_{\mu_{p} v_{p}}^{m_{p}} P_{\mu_{1}} P_{v_{1}} \ldots P_{\mu_{p}} P_{v_{p}}\left(P^{2}\right)^{q}
$$

By commuting $P_{\mu}$ with $\prod^{n}$, we get $\left[P_{\mu}, \prod^{n}\right] \sim \prod_{\mu v}^{n-1} P_{v}+\ldots$ Terms with such factors are not present in the right hand side of (8.6). To compensate such a term with some other ones is also not possible (the compensating term should have the same structure with respect to the generators of translations and therefore there is no such choice). This means that $n_{1}=\ldots=n_{k}=0$. Analogously $\left[P_{\mu}, \prod_{\tau \sigma}^{m}\right]$ $\sim \prod_{\mu \tau}^{n} \prod_{\sigma \gamma}^{m-n-1} P_{\gamma}+\ldots(m \neq 1)$. One might try to compensate this term by a term proportional to $\prod^{m-n} \prod_{\mu \sigma}^{n}$, but as we have just seen, it is impossible to include such terms. Thus (8.5) is possible only in the case of $C_{n}=0$ for $n>0$. One can conclude that the rational invariants, if they exist, are constructed only out of the Casimir operators of the corresponding subgroup $\operatorname{ISO}(p, q)$.

Let us show that there exist invariant operators for the Weyl group, i.e. the Casimir operators $C_{i}$ of $\operatorname{ISO}(p, q)$ group are relative invariants of the dilatation generator. In order to find commutators of $D$ and $C_{i}$ we use the fact that the Casimir operators of $\operatorname{ISO}(p, q)$ group are homogeneous functions of $P$ (which can be proven by constructing $C_{i}$ by the method of expansion as in Sect. 5 and Ref. 12). Therefore, by taking into account (8.1) one can check that

$$
\left[D, C_{i}\right]=m_{i} C_{i}, \quad i=1, \ldots,\left[\frac{p+q+1}{2}\right],
$$

where $m_{i}$ is the power of homogeneity with respect to $P_{\mu}$.

Thus from any two Casimir operators $C_{i}, C_{j}$ of the $\operatorname{ISO}(p, q)$ group, after taking them to necessary powers $k_{i}, k_{j}$ (so, that $k_{i} m_{i}=k_{j} m_{j}$ ), we can obtain a rational invariant of the $\mathrm{W}(p, q)$ group.

Algebraically independent invariants will be one less than the Casimir operators $C_{i}$ of the corresponding $\operatorname{ISO}(p, q)$ group. For instance, for $C_{i}$ $\left(i=1, \ldots,\left[\frac{p+q+1}{2}\right]-1\right)$ one can take as denominator the necessary powers of the 
remaining $C\left[\frac{p+q+1}{2}\right]$; and any rational invariant is obtained from this set by a series of algebraic operations.

Since, according to what has been said above, there are no polynomial invariants for the Weyl group, the number of invariant operators is equal to

$$
\tau_{\mathrm{o}(\mathrm{W}(p, q)}=\left[\frac{p+q-1}{2}\right] .
$$

From (8.7) it follows that, for example, the Weyl group in two-dimensional space does not have any invariants, while the group W(3) has a rational invariant which can be written as

$$
C=\frac{P^{2}}{(\bar{M} \cdot \bar{P})^{2}}
$$

according to Sect. 5 .

Finally, we would like to mention that the methods for constructing the invariant operators of the inhomogeneous groups presented in this paper might be useful also for the case of supersymmetric models [21]: for the supersymmetric theories, the part with commutation relations would correspond to the commutation relations of the homogeneous part of an inhomogeneous group, while the part with anticommutation relations would correspond to the remaining commutation relations of the inhomogeneous group.

\section{References}

1. Racah, G.: Group theory and spectroscopy. CERN report 61-68 (1961); Ergeb. Exakt. Naturw. (Springer Tracts in Modern Physics) 37, 28 (1965)

2. Perelomov, A.M., Popov, V.S. : Casimir operators for U(n) and SU(n). Sov. J. Nucl. Phys. 3, 676, and Casimir operators for the orthogonal and symplectic groups. 819 (1966)

3. Kadyshevskii, V.G., Todorov, I.T.: The ISL(6) symmetry group: Representations and invariants. Sov. J. Nucl. Phys. 3, 96 (1966)

4. Mirman, R. : Invariants and scalars of compact inhomogeneous unitary algebras. J. Math. Phys. 9, 39 (1968); Wong, M.L.F., Yeh, Hsin-Yan: J. Math. Phys. 20, 247 (1979); 22, 935 (1981)

5. Kibble, T.W.B.: Lorentz invariance and the gravitational field. J. Math. Phys. 2, 212 (1961)

6. Utiyama, R. : Invariant theoretical interpretation of interaction. Phys. Rev. 101, 1597 (1956)

7. Hehl, F.W., von der Heyde, P., Karlick, G.D., Nester, J.M.: General relativity with spin and torsion: foundations and prospects. Rev. Mod. Phys. 48, 393 (1976)

8. Stelle, K.S., West, P.C.: Spontaneously broken de sitter symmetry and the gravitational holonomy group. Phys. Rev. D21, 1466 (1980)

9. Gel'fand, I.M. : Mat. Sb. (in Russian) 26, 103 (1950)

10. Beltrametti, E.G., Blasi, A.: On the number of Casimir operators associated with any Lie group. Phys. Lett. 20, 62 (1966)

11. Abellanas, L., Martinez Alonso, L.: A general setting for Casimir invariants. J. Math. Phys. 16, 1580 (1975)

12. Rosen, J.: Construction of invariants for Lie algebras of inhomogeneous pseudo-orthogonal and pseudo-unitary groups. J. Math. Phys. 9, 1305 (1968)

13. Nagel, J., Shah, T.: Expansion of the inhomogeneous symplectic Lie algebras $\mathrm{T}(2 n)$ 午 $\mathrm{Sp}(n)$ to Sp $(n+2)$. J. Math. Phys. 11, 1483 (1970); see also Gilmore, R. : Rank 1 expansions. J. Math. Phys. 13, 883 (1972)

14. Gilmore, R.: Lie groups, Lie algebras, and some of their applications. New York: Wiley 1974 
15. Zhelobenko, D.P.: Compact Lie groups and their representations. Providence, RI: Am. Math. Soc. 1973

16. Dickson, Z.E.: Ann. Math. Ser. A2 25, 294 (1924)

17. Generally speaking, there are still two more sufficient conditions for the existence of only polynomial invariants, namely: i) the nilpotentness of the group, and ii) semi-simplicity. The groups we are investigating here do not satisfy any of these conditions

18. For part of the commutation relations used in this paper, see $[14,19]$

19. Barut, A.O., Raczka, R.: Theory of group representations and applications. Warszawa: Polish Scientific Publishers 1977

20. Weyl, H.: The classical groups. Princeton, NJ : Princeton, University Press 1946

21. Fayet, P., Ferrara, S.: Supersymmetry. Phys. Rep. 32C, 249 (1977)

Communicated by H. Araki

Received March 9, 1982; in revised form March 29, 1983 